SOUTHERN ILLINOIS UNIVERSITY - CARBONDALE, IL 62901

Regulation of Alcohol Permentation by Eacherichia coll

DOE/EFV $1394 I--2$

DE92 016870

David P. Clark, Department of Microblology

The purpose of this profect is to elucidate the way in which the fermentative synthesis of ethanol 18 regulated in the facultative anaerobe Eschertchia col1. We are also Investigating the control of other genes required for fermentation and anaerobic growth. We have 1solated both Btructural and ragulatory mutations affecting the expression of alcohol dehydrogenase, the enzyme repsonstble for the final scep in alcohol synthesis. Some of these regulatory autations also affect other anaeroblially induced genes. The adh gene has been cloned and sequenced. The ADH protein to one of the largest highly expressed proteins in $\underline{\mathrm{E} . \text { coll }}$ and requlres apyroximately $2700 b p$ of UNA for 1 ts coding sequence. We have also isolated mutations affecting the fermentative lactace dehydrogenase. In consequence it is now posstble to construct $\mathrm{E}$. coll strains defective in the productin of any one or more of thelr normal fermentatin products (i.e. formate, acetate, lactate, ethanol and succinate). The factors affecting the ratio of fermentatino products are being investigated by in vivo NMR spectroscopy. Examination if our collection of anaeroblcally controlled gene fustons has shown that many can be switched on in air upon treatment with cyanide or certain other inhibitory agents. We are investigating the basis for this effect at present.

\title{
DISCLAIMER
}

This report was prepared as an account of work sponsored by an agency of the United States Government. Neither the United States Government nor any agency thereof, nor any of their employees, makes any warranty, express or implied, or assumes any legal liability or responsibility for the accuracy, completeness, or usefulness of any information, apparatus, product, or process disclosed, or represents that its use would not infringe privately owned rights. Refer. ence harein to any specific commercial product, process, or service by trade name, trademark, manufacturer, of otherwise does not necessarily constitute or imply its endorsement, resommendation, or favoring by the United States Government or any agency thereof. The views and opinions of authors expressed herein do not necessarily stace or reflect those of the United States Covernment or any agency thereof.

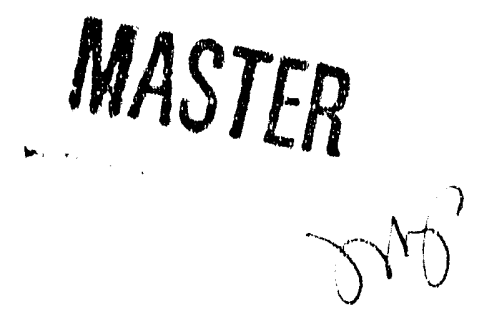




\title{
PROGRESS REPORT
}

for Grant DE-PGO2-88ER13941

\section{REGULATION OF ALCOHOL FERMENTATION IN ESCHERICHIA COLI}

Principal Investigator: David P. Clark

\author{
Department of Microblology \\ Southern Illinols University \\ Carbondale, IL 62901 \\ for the perfod July, 1988 to June, 1989
}

This progress report includes the following manuscripts which are in varfous stages of completion. All are attributable to DOE funding. Some of the recently published papers were included in the form of "submitted manuscripts" in previous report.

1. Reams, S.G. and D.P. Clark (1988) Glucose repression of anaerobic genes of Escherichia col1 is independent of cycl1c AMP. FEMS Letters 56 231-236.

2. Mat-Jan, Fo, Alam, R.Y., and D.P. Clark (1989) Mutants of Escher1ch1a coll deficient in the fermentative lactate dehydrogenase. J Bactertol $171342-348$.

3. Mat-Jan, F., Williams, C.R., and D.P. Clark. Anaeroblc growth defects resulting from gene fusions affecting succinyl-CoA synthetase. Mol. Gen.

Genet. In press.

4. Gupea, S., and D.P. Clark. Derlvarlves of Escherlchla coll lacklng both alcohol dehydrogenase and phosphotranscetylase grow anaerobical. Iy by Lactate fermentation. Submitted to J. Bacteriol.

5. Clark, D.P. The fermentation pathways of Escherlchla coli. PEMS 
Microblology Reviews. Review submitted at edfors request.

The review (\#5 above) summarlzes much of our work over the last few years and includes a brlef description of some recent, unpublished observations. A. The adhE gene gequerice

In collaboration with Dr. J. Parker of this department we have sequenced the adhE gene (Figure 1). A sequence of 2673 bases coding for a protein of 891 amino acids was found between positions 1035 and 3708 of the sequenced region of the adhE plasmid pHIL8. Within this sequence an inverse open reading frame was found which codes for a possihle protein of fust over 400 amino acids (see E1gure 2).

The regulatory sequences for anaerobic expression of the adhE gene are not on plasmid pHIL8. They are presently being subcloned from the previous plasmid pHIL5 which carries a listger segment of chromosomal DNA.

A computer homology search 18 in progress and a manuscript describing the cloning and sequencing of the adhE gene is in preparation.

\section{B. Fermenation products}

We have used in vivo NMR spectroscopy of anaerobic culcures to analyse the rixture of fermentation products. A series of mutants affecting fermentation and constructed strains carrying several mutations have been surveyed (see manuscripts \#2,4, and 5). As expected mutations in adhE abolish the production of ethanol, those in ldha abolish lactate and pta/ack wutants lack acetic acid. More interestingly double mutants in adhe and pra produce no ethanol or acetate but do grow anaerobically on glucose by what is essentially a homolactic fermentation (see 14 ). The critical 1ssue (discussed In detail in ray review 非) is the question of redox balance. Reducing equivalents produced during glycolysis must be consumed during fermentation. The proportions of ethanol (highly reduced) lactate (medium reduced) and acetate (no consumption of reducing equivalents) vary in resnonse to the level 
of reduction of the fermentation substrate. Wth sugar alcohols eg sorbitol, which are more reduced than glucose, the proportion of ethanol is higher whereas with sugar acids (eg gluconic acid, glucuronic acld) the amount of ethanol is less than for glucose. We have preliminary evidence that the hydrogen released from formate by formate hydrogen lyase may be recycled and used to increase the yield of ethanol under some conditions.

\section{Regulation of anserobic genes}

We previously found that some anaeroblcally induced gene fusions may be switched on in air by treatment with cyanide or 5-methoxyindole-carboxylic ac1d (MICA). We introduced mutations in both cytochrome o (cyo) and cytochrome d (cyd) into some of these gene fusion strains and found no aerobic derepression. Furthermore, addition of cyanide still derepressed these anaerobic genes even in a ço cyd double mutant background, despite the fact that the two cytochromes which are the main target slte for cyanide were absent. Analagous results were found with MICA. These agents are therefore derepressing anaerobic genes by some mechantsm other than their previously known inhtbition of resplratory metabolism. Genes which are affected by cyanide and MICA include adh, hyd B and ant (both parts of the formate hydrogen lyase system) and several anaerobically induced gene fusions of unknown function. Several genes involved in anaerobic respiration were tested, but did not respond to cyanide or MICA. The fermentative lactate dehydrogenase was not affected by cyanide or MICA. 
ADH start 1035

stop 3707

$\begin{array}{ll}\text { aa's } & 891 \\ \text { Mw } & 96000\end{array}$

isolechicpoint 6.97 
$10 \quad 20 \quad 30$ GAATTCCGTA TGGCAATGAA AGACGGTGAG GluPhoArg AsnSerVal MetAlaMet IleProtyr

70

TACACCGTTT TyrThrVal

ThrProphe HisArgPhe

130

GATTTCCGGC AspPheArg

IleSerGly

PheProAla

190

GCCTATTTCC

Alatyrphe

Prolleser

LeuphePro

250

GTTTCACCAG

Valsorpro

PheHisGIn

PheThrSer

310

TrpG In *

Iy AAPG IYG IU

LysthrValser

ArgArg * Ala

90
AACTGAAACG

80

TCCATGAGCA

PheHisGlu GInThrGluThr

SerMetSer LysloulysArg

Pro* Ala

Asn * AsnVal

140

AGTTTCTACA

150

GInPheleu HisIleTyrSer

SerPheTyr ThrTyrIleArg

ValserThr

HisI lePheAla

210

TAAAGGGTTT ATTGAGAATA

LeuLysGIy LeuLeuArgI le

* ArgVal Tyr * GluTyr

LysGlyPhe I leGIuAsnMet
270

TTTTGATTTA AACGTGGCCA

Valiouile

Phe * Phe

PheAspleu
* ThrTrpPro

LysArgG I yG In

AsnValAlaAsn
330

40

40

LeuValile TrpaspSer ValHisProCys

GGGATAGTGT

60

Trp * Tyr GlyIleVal PheThrLeuVal

GlyAspMet Gly * Cys Serproloutou

100

110

120

TTTTCATCGC TCTGGAGTGA ATACCACGAC

Pheserser LeuTrpser GluTyrHisAsp

PheHisAr: SerGlyVal AsnThrThrThr

PheileAla Leuglu * IleProArgArg

$\begin{array}{lll}160 & 170 & 180\end{array}$

CAAGATGTGG CGTGTTACGG TGAAAACCTG

GinAspVal AlaCysTyr GlyGluAsnLeu

LysMetTrp ArgValThr ValLysThrTrp

ArgCysGiy ValleuArg * LysProGiy

220

230

240

TGTTTTTCGT CTCAGCCAAT CCCTGGGTGA

Cyspheser SerGinPro IleProgly *

ValPheArg LeuSerGin SerLeuGlyglu

PhePheVal

SerAlaAsn

ProTrpValser

280

ATATGGACAA

IleTrpThr

TyrGIyG In

290

300

CTTCTTCGCC CCCGTTTTCA

Thrserser Propropheser

MetAspasn

LeuLeuArg ProArgPheHis

PhePheAla ProvalPheThr

340

350

360

AGGTGCTGAT GCCGCTGGCG ATTCAGGTTC

ArgCys * CysArgTrp ArgPheArgPhe

ATATTATACG CAAGGCGACA

ProTrpala AsnileIle ArgLysalaThr

HisGlyGin IleLeuTyr AlaArgArgGin

MetGlyLys TyrTyrThr GinGlyAsplys

GIyAlaAsp

AlaAlagiy AspSerglyser

ValleuMet

ProleuAla

I leG InValHis

\section{0}

ATCATGCCGT

I lekietPro

SerCysArg

380

390

400

410

420

TTGTGATGGC TTCCATGTCG

PheValMet AlaserMetSer

GCAGAATGCT

AlagluCys

GInAsnAla

TAATGAATTA

LeuMetAsn

HisAlaVal

CysAspgly PheHisvalgi

Argmetileu

Asng Iule

ThrThrValleu

AsngluLeu

GInGInTyrCys

430

440

450

460

470

480

TTTAAGGCAG TTATTGGTGC CCTIAAACGC

PhelysAla Valilegiy AlaleuLysArg

GlyArgAla GlyArzAsnPhe
AlaglyArg GlyValilePhe

AlaMetser

LeuArgG In

LeuleuVal ProleuAsnala

AspgluTrp GInGlyGly Ala * PhePhe

* Glyser

TyrTrpCys

Pro * ThrPro

$490 \quad 500$

510

CGCCTGAATA

CTGGTTGCTA

LouValala

ThrProglu

TrpleuLeu

ArgLeLASn

GlyCysTyr

Ala* Ile

AGTGATAATA

* ValileIle

Lys * **

SerAspasnLys

520

530

540

AGCGGATGAA TGGCAGAAAT TCGAAAGCAA

SerGly * MetAlaglu IleArgLysGin

AlaAspglu TrpGinlys PheGluserlys

Argketasn

GIyArgAsn

SerLysAlaAsn

$550 \quad 560$

570

580

590

800

ATTCGACCCG

I leArgPro

GTCGTCGGT'

CAGGGCAGGG

TCGTTAAATA

SerleuAsn

PheAspPro

G lyArgArg

PheArgAlagly

Arg*Ile

GCCGCTTATG

SerArgleu

AlaAlatyr

Proleuket

TCTATTGCTG

Cysleuleuleu

ValTyrCystrp

SerileAlagly 
CysAsparg Valleuleu LysCysLeuArg Leuproval Tyr * Leu ProGluAlaval

ValthrVal Cyspheser AsnAla * Gly

$670 \quad 680 \quad 690$

CCAGTTTGCT CAGGCTCTCC CCGTGGAGGT

ProValCys SerGlySer ProArgGlygly

GinPheAla GinAlaleu Provalgluval

Serleuleu Argleuser ProTrparg *

* Procys

Alasergin MetProgluala

$700 \quad 710 \quad 720$

AATAATTGAC GATATGATCA TTTATTCTGC

Asnasn * ArgTyrasp HisleupheCys

IleIleAsp AspMetIle IleTyrSerAla

* LeuThr Ile * Ser PheIleleupro

730

740

750

CTCCCAGAGC CTGATAAAAA CGGTTAGCGC

LeuProGlu

SerGinser LeuIlelys ThrValserala

ProArgala

790

* * Lys

Argleualaleu

800

810

GGTAGCCAGC AGCATCTGCG ATGCAGATCA

GlySerGIn

Valalaser

* ProAla

GlnHisLeu

SerIlecys

ArgCysArgSer

AspalaAspH is

Alaserala

MotGlnIleThr

850

GCTTACGCCA

Alatyrala

LeuThrPro

LeuArgH is

860

870

CCTGGAAGTG

ThrTrplys

Progiyser

Leugluval

ACGCATTAGA

* Argilearg

Aspalaleuglu

Thritis * Arg

910

920

920

930

GTGAGTGTGA GCGCGAGTAA

ValAsnH is

* IleThr

LysSerGin

SerGlucys

Valserval

GluArgGlu *

Serajaserlys * Val*

AlaArgValser

990

970

TCACCGCACT

SerProHis

HisArgThr

Thralaleu

980

GACTATACTC

* LeuTyr Serargilearg

AspTyrThr LeuValpheGlu

760

TTCGTTAATA

PheValasin

Serleulle

Arg * Tyr

820

CGTAATCAGT

Argasng In

Vallieser * SerVal

880

GATAATAACT

Aspasnasn

I leI leThr

* * Leu

770

780

CAGATGTAGG TGTTCCACAG

ThrAspVal GlyValProGln

GlnMet * ValPheHisArg

ArgCysArg CysSerThrGly

830

840

ACCCAGAAGT GAGTAATCTT

TyrProglu ValserAsnLeu

ThrGinlys

* Valileleu

Glu * SerCys

890

900

CTAATGTTTA

SerAsnVal

LeuMe tPhe

* Cysleu

940

GCTTTTGATT

l PhoAsT

950

TTCATAGGTT

PheHisArg

LeuleuIle PheIlegly

Phe * Phe

Ser * Val

AACTCTTTTA

* Thrleuleu

Lysleuphe *

AsnSerPheSer

1,010

1,000

GCAGATGATT TACTAAAAAA

AlaAspasp LeuLeulys

Tyr * Lys

GinMetile

Thrlyslys

1,060

1,050

1,030

1.,040

SerTyrSerser

Arg * Phe

1,070

TAACGCACTC

TCGCTGAACT

SerLeuAsn

Arg * Thr

Alagluleu

LeuThrH is

er * SerVal

I leArgArg

AlaleuTrp LeuLeuleuMet

SerGlyglu

HisTyrGly

CysTyr * Cys

GInGluSer

Ilethetala

ValThrAsnVal

1,090

1,100

1,110

TAAAAAAAGC

CCAGCGTGAA

TATGCCAGTT

* LysLys

Proserval

LyslysSer ProAla*

Lyslysala Ginargilu

AsnHetProVal

IleCysGinPhe

TyrAlaserphe

1,120

Asnalaleu

Argargalacys

AAGCAAATCA

LeuSerLysSer

* AlaAsnHis

LysGinIleI le

1,020

GTTTAACATT

LysPheAsnI le

SerLeuThrleu

Yal * HisTyr

1,130 GCAAGTAGAC

Serleulys Serlys * ThrlysSerSer

erleulys Serlys * ThrlysSerSer

HisSerArg AlaSerArg GlnAsnLeupro ThrGinglu GinValAsp

LysI lePheArg

$1,150 \quad 1,160 \quad 1,170$ GCGCCGCCGC TCTGGCTGCT GCAGATGCTC

Alapropro LeuTrpleu Leuglnketheu

ArgArgarg SerGlyCys CysArgCysSer

AlaAlaAla LouAlata AlaAspalaArg

1,180

1,190

1,200

GAATCCCACT CGCGAAAATG GCCGTTGCCG

GluSerHis SerArglys TrpProLeuPro AsnProThr ArgGluAsn GIyArgCysArg 
1,210 GGGTATCGTC GAAGATAAAG
AATCCGGCAT

AsnProAla TrpValser Serlysilelys

IleArgHis GiyTyrArg ArgArg * Ser

SerGiymet GlyIleVal GluAspLysVal
$1,270 \quad 1,280 \quad 1,290$

TCTACAACGC CTATAAAGAT GAAAAAACCT

SerThrThr ProIlelys MetLyslysPro

Loug InArg Leu * Arg

TyrAsnAla TyrLysAsp
* Lysasnleu GlulysThrCys
1,240

TGATCAAAAA

ThrThrieu LouleuAsnIle Aspginlys ProLeuCys Phe * IleTyr

1,260

TCTGAATATA
$1,330 \quad 1,340 \quad 1,350$

GTACCATCAC TATCGCTGAA CCAATCGGTA

Valproser LeuSerlou AsnginSerVal

TyrHisHis TyrArg *

ThrIleThr IleAlagiu
ThrAsnArgTyr

Prolleglyile
1,300

GTGGTGTTCT

Valvalphe

TrpCyssor

GIyValLeu
HisPheAla

1,310

GTCTGAAGAC

Cysleulys

$\mathrm{Vel} * \mathrm{Arg}$

SerGluAsp

Serglutyrile

1,320

GACACTTTTG

ThrThrleuleu

ArgH isPheTrp

AspThrPheGly

$\begin{array}{rrr}1,360 & 1,370 & 1,380\end{array}$

TTATTTGCG TATCGTTCCG ACCACTAACC

LeuPheala ValserPhe ArgProleuThr

TyrLeuArg TyrargSer AspHis * Pro

IleCysGly IleValPro ThrThrAsnPro

$1,390 \quad 1,400 \quad 1,410$

CGACTTCAAC TGCTATCTTC AAATCGCTGA

Argleugin LeuleuSer SerAsnArg *

AspPheAsn CystyrLeu GinIleAlaAsp

ThrSerThr AlaIlePhe LysSerleuIle

1,450

TCTCCCCGCA

SerProArg

LeuProAla ProAlacys LysArgCysh is

SerProHis ProArgala LysAapalaThr

1,510

TGCTATCGC

LeuLeuSer

CysTyrarg

AlaIleAla
1,570

AACTGTCTAA

AsnCysleu

ThrVal *

LeuSerAsn

1,630

CGGGCATGGT

ArgAlaTrp

GlyHisGly

GlyMetVal

1,690

ACACTCCAGT

ThrLeugin

HisserSer

ThrProval

1,750

TGTCCAAAAC

CysProlys

Valginasn

Serlysthr

$$
1,520
$$

TGCCGGTGCT

Leuproval

CysArgCys

A lagly y a

1,580

CGCACTGATG

ThrHis *

ArgThrAsp

Alaleuket.

1,640

TAAAGCCGCA

LeuLysPro

* SerArg

LysalaA a

1,700

TGTTATCGAT

Leuleuser

CysTyrarg

YaII leAse

1,760

CTTCGACAAC

ProserThr

LeuArgGin

PheAspasn
1,530 CCGAAAGATC LeuArgLysIle SerGluArgSer

Prolysaspleu

1,590

CACCACCCAG

CysThrThrGIn

A laproproArg

$\mathrm{HisH}$ isProAsp

1,650

TACAGCTCCG

HisThrAlapro

I leg inleuArg

TyrSerSerGiy

1,710

GAAACTGCTE

Metilyslauleu * AsnCys * GluThrAlaAsp

1,770 GGCGTAATCT Thrala * Ser Argargasnleu GlyvalI leCys
1,420

TCAGTCTGAA

Serval *

GInSerglu

Serleulys

1,480

CCAACAAAGC

ProThrlys

GInGInSer Asnlysala

1,540

TGATCGGCTG

* Serala

Aspargleu

I leGiyTrp

1,800

ACATCAACCT

ThrSerThr

HisGlnFro

I leAsnLeu

1,660

GTAAACCAGC

Valasngla

* ThrSer

1,430

GACCCGTAAC

ArgProVal

AspPro *

ThrargAsn

1,490

GGCTGATATC

Argleul le SerpheCysarg

Gly * Tyr Argseralagly

AlaAspi lo Valleuginala

GATCGATCAA CCTTCTGTTG

GlySerIle AsnLeuleuleu

Aspargser ThrPheCys *

IleAspgin ProSerValgiu

1,610

1,620

GATCCTCGCG ACTGG 2 GGTC

* Serser ArgleuValval

AspProArg AspTrpTrpSer

I leleuala ThrGlyglyPro

1,670

1,680

TATCGGTGTA GGCGCGGGCA

LeuSerVal * AlaArgala

TyrArgCys ArgArgG IyGin
LysProAla IleglyVal GlyAlaglyasn

1,720

1,730

1,740

ATATCAAACG TGCAGTTGCA TCTGTACTGA

IleserAsn Valginleu HisleuTyr *

TyrGinThr CysSerCys IloCysThrAsp

Ilelysarg Alavalala Servalieuket

1,780

1,790

1,800

GTGCTTCTGA ACAGTCTGTT GTTGTTGTTG

Valleuleu Asnserleu Leuleuleuleu Cysphe * ThrValCys CysCysCys * Alaserglu Glnserval ValvalvalAsp 
1,810

ACTCTGTTTA

ThrLeuPhe

LouCysteu SerValTyr

1,870

GTAAAGAGCT

VallysSer

* Argala

Ly yG IuLeu
1,820

TGACGCTGTA

MotThriau

* ArgCys Thr * ThrPhe

AspalaVal
1.830 CGTGAACGTT

TyrValAsnVal $\begin{array}{rr}1,840 & 1,850 \\ \text { TTGCAACCCA CGGCGGCTAT }\end{array}$

LeuGinPro ThrAlaAla

CysasnPro ArgArgLeu

AlathrHis
GlyGlyTyx

1,860

CTGTTGCAGG

I loCysCysArs

SerValAlagly

LouLougInG Iy

1,890

GAAAGCTGTT CAGGATGTTA

* Lysleu PheArgMetleu

GluserCys SerGlyCysTyr

GInAspVali le

1,930

SerleuVal

ArgTrpSer

ValGIyGIn

1,990

CCAAGATTCT

ProArgithe

GInAspSer

Lysi leleu
1,950

GCCAGCCTAT AAAATTGCTG

SerGinfro I lelysteuleu

Alaserleu * AsnCys *

ProAlaTyr

2,000

GATCGGTGAA

* Serval

Asparg *

I leGlyGlu

LysI le A laGlu
2,010

GTGACCGTTG

Lys * ProLeu SerAspargCys

ValThrValVal
1,900

TCCTGAAAAA

Ser * Lys

Proglulys
Leulysasn

1,810

CGGTGCGCTG

ThrValarg

ArgCysala

Glyalaleu
1,920

AACGCGGCTA

* Thrarglou

G IuArgG IyTyr

AsnalaAlalle
2,050

AACTGTCCCC

AsnCysPro

ThrValpro

LeuSerPro
2,060

GACTCTGGCA

ArgLeuTrp

AspSerGly

ThrLeuAla
1,960

1,970

AACTGGCAGG

AsnTrpGln

ThrG IyArg

LeuAlaGIy
CTTCTCTGTA

Alaserlou

str.

Pheserval ProgluAsnThr

2,030

2,040

TTGATGAAAG CGAACCGTTC GCACATGAAA

LeuHetlys AlaAsnArg SerHisMetLys * * Lys ArgthrVal ArgThr * Lys

AspGluSer

Gluprophe

AlaHisGluLys

2,070 ATGTACCGCG GinCysThrAla AsnValproArg MetTyrArgA. a
2,080 CTAAAGATTT

Leulysile

* ArgPhe LysAsp?he
2,090

CGAAGACGCG
2,100
ArgArgArg GlyArglysSer

GiuAspala Valgiulysala
2,110

CAGAGAAACT GInArgAsn

ArgGluThr

Giulysleu
2,120

GGTTGC'RATG

GGTTGC'TATG

GlycysTyr

valAlamet
2,130 GGCGGTATCG TrpAlaValser GIyArgTyrArg GlyGlyIleGly
2,140 GTCATACCTC

Valilepro

SerTyrLeu HisThrSer
2,150

TTGCCTGTAC

LeuAlacys
Cysleutyr

\section{ACTGACCAGG}

2,170

AT AACCAACC

IleThrAsn

* Prothr

AsnG InP ro
2,180

GGCTCGCGTT

Argleuala

GlySerArg

AlaArgVal
2,190

TCTTACTTCG

PheleuThrser

PheleuteuAr

SerTyrPheGIy
2,200

GTCAGAAAAT

ValArgLys

SerGIuAsn
2,250

2,240

AGCGTCTCAG

GInArgLeu

Servalser

Alasergln
GGTGGTATCG

ArgValValser

GIyTrpTyrArg

GIyG IyI leG Iy
2,260

GTGACCTGTA

ValThrCys * Proval Aspleutyr
2, 210

His * Progly

ThrAspG InAsp

2,220

* HisPro

AsnThrero

2,300

2,310

2,290

CCCTGACTCT

Pro * Leu GGGTTGTGGT TCTTGGGGTG

TrpValVal ValleuglyVal

ProAspSer GlyLeuTrp PheLeuGlyTrp

LeuThrleu GIyCysGly SerTrpglygly

2,320
GTAACTCCAT
ValThrPro
* LeuHis
AsnserIle

2,370

2,350

2,360

2,380

AACACCTGAT CAACAAGAAA ACCGTTGC'TA
AsnThr * SerThrArg LysProLeuLeu

ThrProAsp

GInGInGIu AsnArgCys *

HisleuIle Asniystys

ThrValAlaLys

AGCGAGCTGA

SerGluLeu

Alaser *

ArgAlaglu.

GAAAACGGCG CGTATCCTGA

* Lysarg ArgValser *

GluAsngly AlatyrProAsp

Lysthrala ArgileLeuIle

2,270

2,280

TAACTTCAAA CTCGCACCTT

IleThrSer AsnSerHisleu

* Leugir ThrArgThrPhe

AsnPhelys LeuAlaProser.

$2,330 \quad 2,340$

CTCTGAAAAC GTTGGTCCGA

Serleulys ThrleuValarg

Leu * Lys ArgTrpSerGiu 
2,410

TTCCGAAATC

PheArgAsn

SergluIlo

Prolysser

2,470

CTGATGGCCA

LeuMetAla

* Trpero

Aspg IyH is

2,530

CTGATCAGAT

LeUI leArg

* SerAss

Aspginile

2, 590

TAGAAGCGGA

* Lysare

ArgSergly

GluAlaAsp

2,650

AACCAGACGT

AsnGInThr

ThrArgArg

ProAspVal

2,710

GGGTTATGTA

GlyleuCys

GlyTyrVal

ValmetTyr

2,770

TCCGTAAACG

SerValasn

Pro * Thr

ArgLysarg

2,830

CCACCACTTC

Proproleu

$\mathrm{HisH}$ is $\mathrm{Phe}$

ThrThrSer

2,890

CTGGTCAGAA

LouValarg

TrpSerGiu

GIyGInLys

2,950

CAACCTGGT

ProThrTrp

GInProGiy

AsnLeuVal
2,420

TATCTACTTC CGCCGTGGCT

Lousorthr SorAlavalala

TyrLeulou ProproTrplou

I le'TyxPhe

ArgArgGlyser

2,480

2,480

CAAACGTGCG CTCATCGTGA

ThrasnVal

ArgSerSer *

GInThrCys AlaHisArgAsp

LysArgala

LeuI lovalThr

2,540

2,550

CACTTCCGTA CTGAAAGCAG

SerLeupro Tyr * LysGin

HisPheArg ThrGluSerSer

ThrServal LeulysalaAla

2,600

CCCGACCCTG

ThrargPro

ProAspPro

ProThrleu

2,610

AGCATCGTTC

* Alaserphe

GluHisArgSer

SerIleValarg

2,660

2,670

GATTATCGCG CTGGGTGGT

* Leuser argTrpValval

AspTyrarg AlaglyTrpTrp

I loI leala LeuGlyglygiy

2,720

2,730

CGAACATCCG

Thrasnile ArglysLeuThr

ArgThrSer

GluH isPro

GlyAsnSerLeu

GluThrHisPhe

2,780

2,790

TATCTACAAG TTCCCGAAAA

ValSerThr SerSerArglys

Tyrleugln ValprogluAsn

IleTyrLys

PheProlysMet

2,840

2,850

TGGTACAGGT TCTGAAGTCA

Leuvalgin Valleulysser

TrpTyrarg Phe * SerHis

GIyThrGly SerGluValThr

2,900

2,910

ATATCCGCTG GCAGACTATG

AsnIleArg TrpGInThrMet

Ileserala GiyargLeuCys

AlaAsptyrala

2,970

2,980

TATGGACATG

CysargSerPro

TyrglyHis AlagluValpro

MetaspMet

ProlysSerleu
2,440 CCCTGCCAAT

ProCysGin

ProAlaAsn

LouProI lo

$2,500 \quad 2,510$

CTGACCGCTT CCTGTTCAAC

LeuThrala

SerCysser

* Proleu ProValgin

AspargPhe LeuPheAsn

CGCGCTGGAT

SOIARgTrP

ArgAlagly

GAAGTGATTA

* SerAsptyr

Gluvali leThr

2,560

CAGGCGTTGA

GinAlaleu

ArgArg *

GIyValglu

2,620

GTAAAGGTGC

VallysVal

* ArgCys

2,570

AACTGAAGTC TTCTTCGAAG

LysLeulys SerSerSerlys

Asn * Ser LeuLeuArgSer

ThrGluVal PhopheGluVal

Arg'ThrGly LysLeuleuGln

LysGiyala GiuleuAla Asnserphelys

2,630

2,840

AGAACTGGCA AACTCCTTCA

GlnAsnTrp GinThrProSer

2,890

2,700

GTTCCCCGAT GGACGCCGCG AAGATCATGT

ValProArg TrpthrPro ArgArgSerCys

PheProAsp GlyArgArg GluAspHisVal

Serprollot AspalaAia LysileMetTrp

2,740

2,750

2,760

TCGAAGAGCT GGCGCTGCGC TTTATGGATA

SerlysSer TrpargCys AlaleuTrpIle

ArgArgala GlyAlaAla LeuTyrglyTyr

Glugluleu AlaLeuArg

PheMetAspIle

$\begin{array}{r}2,800 \quad 2,810 \\ \hline\end{array}$

TrPAl $*$ AGCGAAAAT

GlyArgGlu SerGluAsn

GiyVallys Alalysmet

2,860

2,870

CTCCGTTTGC GGTTGTAACT

LeuArgleu Argleu *

SerValCys GiyCysAsn

PropheAla ValvalThr

* ArgargTyr

AspaspalaThr

2,920

2,930

2,940

CGCTGACTCC GGATATGGCG ATTGTCGACG

Arg * Leu ArgIleTrp ArgLeuSerThr

AlaAsper GlyTyrgly AspCysArgArg

LeuThrPro. AspHetAla I leValAspala

2,980

TGTGTGCTTT

Cysvalleu

Valcysphe

CysAlaphe

CGGTGGTCTG

Servalval

ArgTrpser GlyArgSerAsn

GIyGIyLeu

AspalaValThr 
3,010

CTCACGCCAT

LeuThrero

SerArgHis

HisAlaMet

3,070

TGCAGGCACT

CysArgH is

AlaglyThr

GInAlaLou

3,130

TCCGGTAGC

I leArg *

SerGlyser

Provalala

3,190

3,020

3,030

GTTTCTGTAC

Trplyslou HetPheleuTyr

GlyserLou

CyspheCysthr

GIuAlaTyr

ValserValleu

3,080

GAAACTGCTG

* AsnCys

GluThrAla

Lysleuleu

3,140

3,090

AAAGAATATC

* LysasnI le

GIUArgI leser

LysG IuTyrLeu

3,150

GCGTGAACGT

ArgVaIAsn

Ala * Thr

ArgGIUArg

3,200

GGGTGTATGT

TrpValTyr

GlyCyshet

$t$

alThrGint

SerLeuAsngly

GTTCACAGTG

ValpheThrVal

CysSerGInCys

$\mathrm{ValH}$ isSerAla

3,210

ACGCCTTCCT

Argleupro

GIyVaICys

HisserMetAlo

3,260
GGCAAACGCC

3,270
CTGCTGATTT

3,250

roCys * Phe

ArgThrVal

Alatrger

TrpGlnThr

GIyLysArg ProAlaAspleu

HisGIyLeu

AlaAsnAla.

LeuLouI leCys

3,310

3,320

3,330

ACCCGACCAA

ThrArgPro

ProAspgin

ProThrlys

GCAGACTGCA

TTCAGCCAGT

SerArgleu HisserAlaser

AlaAspCys IleGlnProVal

GlnThrAla

PheSerGInTyr

3,370

3,380

3,390

CTGAAATTGC

LeuLysLeu

* AsnCys

CGACCACTTG

ProThrin

Argroleu Glyserglua

GluIleAla AspHisLeu

Gisergiuarg

GlyLeuSerAla

3,450

3,430

3,440

GGCATGGCTG

TrpHisGIy TrpLysArg *

AGAAACTGCT

ArgAsnCys

GluThrAla

Lysleuteu

GIyMetAla

GIyAsnAIaglu

GluThrLeulus

3,510

3,490

GTGAAGCTGG

Vallysleu

* SerTrp

3,500

CGTTCAGGAA GCAGACTTCC

ArgSerGly SerArgLeuPro

GIuAlagly Valgingiu AlaAspiheleu

3,550

CATTCGATGA

HisSermet

IleArs *

3,560

3,570

CCAGTGCACC GGCGCTAACC

ThrSerala ProAlaleuThr

ProvalHis ArgArg * Pro

PheAspAsp
GInCysThr
3,040

3,050

3,060

TGGCATCTGA GTTCTCTGAT GGTCAGGCTC

TrpHislou Serserlou MetValArglou

GlyIle * Valleu * TrpSerGiyser

Alaserglu PheSerAsp GlyglnAlaLeu

3,100

3,110

3,120

TGCCAGCGTC CTACCACGAA GGGTCTAAAA

CysGinArg ProThrThr LysGlyLeuLys

Alaserval LeuProArg ArgVal * Lys

ProAlaser TyrHisGlu GIySerLysAsn

3,160

CAGCGACTAT

GInArgLeu

SerAspTyr

AlaThrile

3,170

3,180

CGCGGGTATC GCGTTTGCGA

SerArgVal SerAxgLeuArg

ArgGlyTyr ArgValCysGIu

AlaGiylie AlapheAlaAsn

3,220

3,230

3,240

CGCACAAACT GGGTTCCCAG TTCCATATTC

ArgThrasn TrpValPro SerSerIlePhe

AlaginThr GlyPhePro ValProTyrSer

Hislysteu

GlySerGin

PhoH is lePro

3,280

GTAACGTTAT

3,290

3,300

TCGCTACAAT GCGAACGACA * Argtyr Serleugin Cysgiuarggin AsnValio Argtyrasn AlaAsnaspasn

3,340

3,350

3,360

ATGACCGTCC GCAGGCTCGC CGTCGTTATG

MetThrVal ArgArgleu AlaValValMet * Proser Alaglyser ProserleuCys

AspargPro GInAlaArg ArgArgTyrAla

$\begin{array}{rrr}3,400 & 3,410 & 3,420\end{array}$

CACCGGGCGA CCGTACTGCT GCTAAGATCG

HisArgAla ThrValleu LeuLeuArgser

ThrGlyArg ProTyrCys Cys * Asparg

ProGiyAsp ArgThrala Alalysileglu

$\begin{array}{rrr}3,460 & 3,470 & 3,480 \\ \text { AAGCTGAACT } & \text { GGGTATTCCG } & \text { AAATCTATCC } \\ \text { LysLeuAsn TrpVEIPhe } & \text { ArgAsnLeuSer } \\ \text { Ser * Thr GlyTyrSer } & \text { GIUIleTyrPro } \\ \text { AlaGluLeu GlyIlePro } & \text { LysSerIleArg }\end{array}$

$3,520 \quad 3,530$

TGGCGAACGT GGATAAACTG TCTGAAGATG

3,540

TrPArgThr TrpIleAsn CysLeulysMet

Glygluarg Gly * Thr Val * ArgCys

AlaAsnVal Asplysieu Sergluaspala

$3,580 \quad 3,590$

CGCGTTACCC GCTGATC'TCC GAGCTGAAAC

3,600

ArgValThr Arg * Ser ProSer * Asn

Alaleupro Alaspleu ArgAlagluThr

ArgTyrPro LeuIleSer GluLeuLysGin 
3,610

AGATTCTGCT

ArgPheCys

AspSerAla

Ileleuleu

3,670

AAGAAGCTGC

LysLysLeu

ArgSerCys

LeuArgLeu

SerGly *

GlualaAla
3,620

TrpIlePro ThrThrValval

GiyTyrLeu LeuArgser * AspThrTyr TyrGlyArgAsp
3,640

ATTATGTAGA

I leMet *

LeuCysar

TyrValglu
3,650 AGGTGAAACT

LysVaILys

Arg * Asn CysSergluglu

GlyGluThr

AlaAlalysiys
$3,730 \quad 3,740 \quad 3,750$

TGTCTGGCAA CATAAACGGC CCCTTCTGGG

CysleuAla Thr * Thr Alaprosergly

ValTrpGin Hislysarg ProLeuleugly

SerGlyAsn IleAsngly PropheTrpala
3,700

AAGCGAAAAA

LysArgLys SerGiuly AsnProLeu AlalysLys
3,710

ATCCGCTTAA

I leArgLeu
3,720

TCAGTAGCGC AsnGin * Arg I leSerSerAla

Servalalaleu
$3,790 \quad 3,800 \quad 3,810$

GATCCTTAAA

AspPro *

Ileleulys

SerLeuAsn
CTGAGGCACT ATAACGGCTT
ThrGluala Leu * Argleu
3,760

3,770

TACTMACC

GinCysarg SerVallys Asp * LeuThr AsnalaAsp GInLeuArg Ileser * P $=0$ HetProIle Ser * Gly LeuValAsparg

$3,820 \quad 3,830 \quad 3,840$

CCACAACAGG GAGCCGTTTT CTTAGTCCAC

ProGlngln GlyalaVal PheleuValHis

HisAsnarg GluProPhe Ser * SerThr

* GlyThr IleThrAlaser ThrThrGly SerArgPhe LeuSerProLeu

$3,850 \quad 3,860 \quad 3,870$

TTCTCAATGA TCTGCTCGAT TTCAGTGACC

Pheserket

Sergln *

LOUAsRAsp

I lecysSer I. IeSerValthr

SeralaArg Phegln * Pro LouLouAsp PheSerAspHis
3,910

TATTTGCAGA

TyrLeuGln IleCysarg PheAlaglu

$$
3,880
$$

ATCCGCTTAT
3,890

GCCTCCGCCC

I leArgleu CysleuArg ProLeulisasn

3,900 SerAlaTyr AlaSerAla LouCysThrThr Proleuket Propropro SerAlaginLeu

3,930

3,920

ACACCTTCCC ACCGAGTGGA

AsnThrPhe ProProSerGly

ThrProSer HisArgValAsp HisleuPro ThrGluTrpIle

3,970 CGACCGTTCG

ArgP roPhe

AspArgSer ThrValarg
3,990

3,980

CCGCCGTCGT

AlaAlaval

roProSer PheThrolysly LeuProG IyAsp
3,940

TACAACACTG

TyrAsnThr

ThrThrLeu GinHisCys

4,000

ACATGGTTAT

ThrTrpleu

HisGlyTyr MetValile
3,950 CCTGACGCTT
3,960
Ala * Arg Pheleuleumet

ProAspala PheCysserCys

$$
4,030
$$

ArgArgAr8
4,050

4,040 ATGTTGTTCG

Aspvalval

Metleuphe CysCysser

CCGTCTGAAC
gArgLeuAsn
ProIleThr

GlnLeuPro

AsnTyrArg

4,090

TGCTGGCCCG

CysTrpPro

$$
4,100
$$

CAGCGCTGTC

AlaAlaleu

GinArgCys

AlaglyPro

LouAlaArg SerAlaVal ThrGinala
Alaval * Thr

Proserglupro

4,110

ACCCAGGCGC

SerProArgAr:

H isProGlyAla
LeuAlaArg SerAlaval ThrGinala
4,060

CTGAGCGCGG

euSerAla Aspglyglu ArgGly * Thr * Alrarg MetalaAsn Giyaspglupro GluArgGly TrpargThr GlythetAsnLeu 


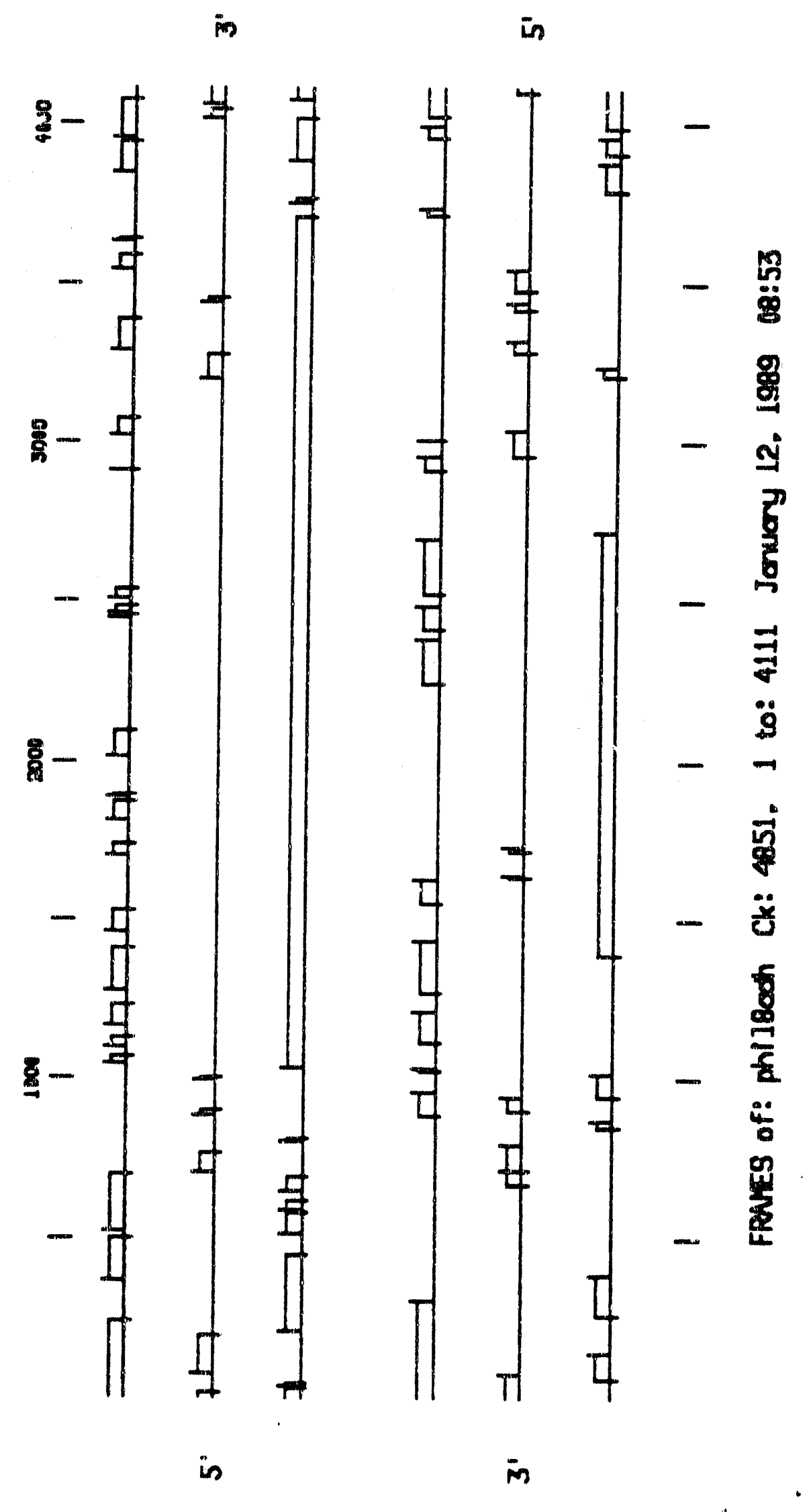



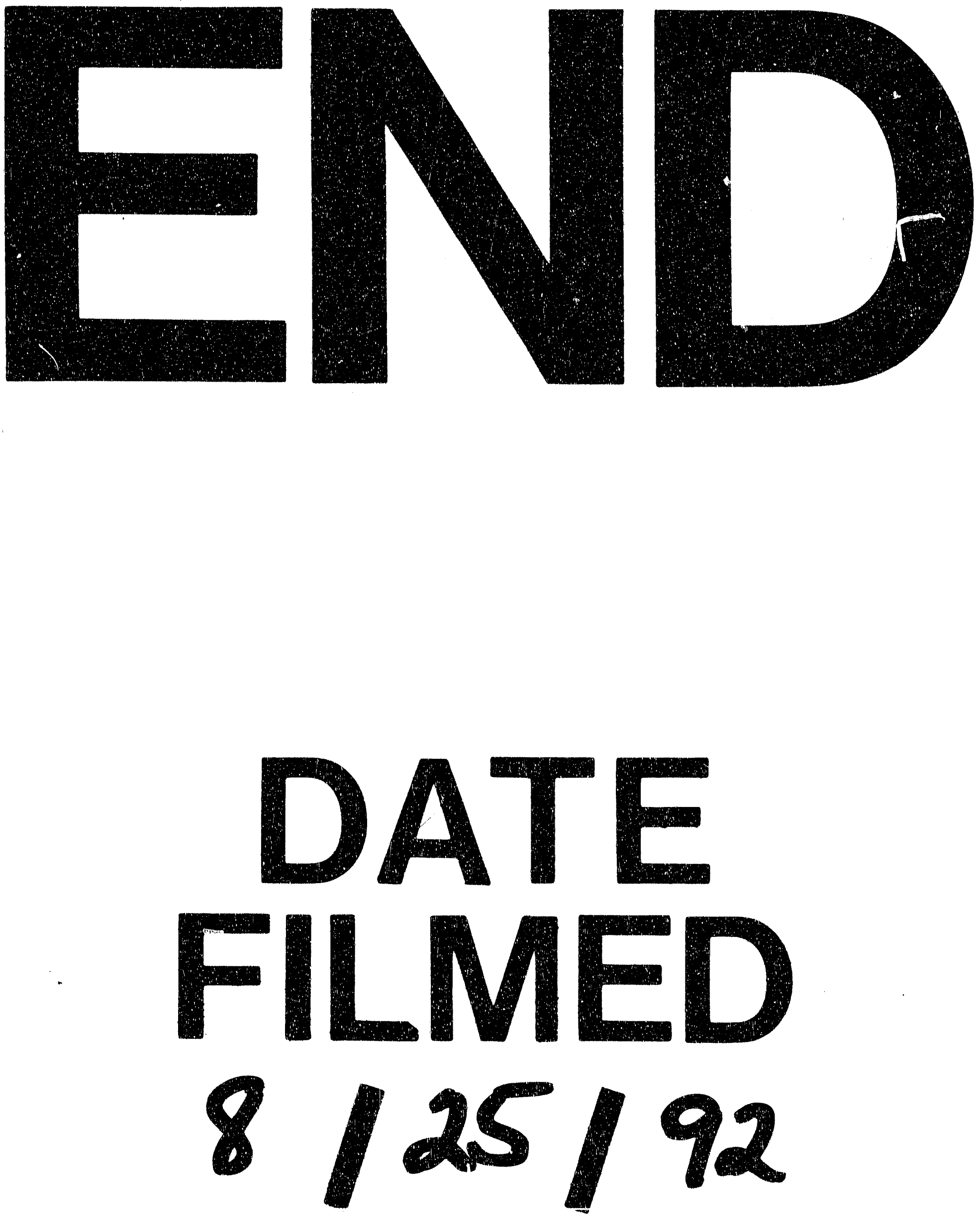
